\title{
Diclofenac Identified as a Kynurenine 3-Monooxygenase Binder and Inhibitor by Molecular Similarity Techniques
}

\author{
Steven Shave, ${ }^{\dagger}$ Kris McGuire, ${ }^{\ddagger}$ Nhan T. Pham, ${ }^{\dagger}$ Damian J. Mole, ${ }^{\ddagger}$ Scott P. Webster, ${ }^{\S}$ \\ and Manfred Auer, ${ }^{*}+\odot$ \\ ${ }^{\dagger}$ School of Biological Sciences, University of Edinburgh, The King’s Buildings, Max Born Crescent, CH Waddington Building, \\ Edinburgh, Scotland EH9 3BF, U.K. \\ ${ }^{\ddagger}$ MRC Centre for Inflammation Research, Queen’s Medical Research Institute, and ${ }^{\S}$ Centre for Cardiovascular Science, Queen's \\ Medical Research Institute, The University of Edinburgh, 47 Little France Crescent, Edinburgh EH16 4TJ, U.K.
}

Supporting Information

ABSTRACT: In this study, we apply a battery of molecular similarity techniques to known inhibitors of kynurenine 3monooxygenase (KMO), querying each against a repository of approved, experimental, nutraceutical, and illicit drugs. Four compounds are assayed against KMO. Subsequently, diclofenac (also known by the trade names Voltaren, Voltarol, Aclonac, and Cataflam) has been confirmed as a human KMO protein binder and inhibitor in cell lysate with low micromolar $K_{\mathrm{D}}$ and $\mathrm{IC}_{50}$, respectively, and low millimolar cellular $\mathrm{IC}_{50}$. Hit to drug hopping, as exemplified here for one of the most successful anti-inflammatory medicines ever invented, holds great promise for expansion into new disease areas and highlights the not-yet-fully-exploited potential of drug repurposing.

\section{INTRODUCTION}

Increasing costs ${ }^{1}$ and attrition rates ${ }^{2}$ associated with bringing a new drug to market have driven interest in repurposing existing drugs for new indications. ${ }^{3,4}$ Routine use of drugs "off-label" is well-documented and most prevalent in pediatrics, with drugs typically approved for use in adults being prescribed by physicians for use in children. ${ }^{5,6}$ With the ability to use existing, approved drugs "off-label", the application of molecular similarity techniques offers an extremely attractive route toward treating disease states, identifying approved drugs capable of making similar interactions to known ligands of target proteins.

Kynurenine 3-monooxygenase (KMO) is a nicotinamide adenine dinucleotide phosphate (NADPH)-dependent flavoprotein hydroxylase that catalyzes the hydroxylation of kynurenine (L-Kyn) to generate cytotoxic 3-hydroxykynurenine (3-HK). ${ }^{7,8}$ KMO has been identified as an important therapeutic target in systemic inflammation triggered by acute pancreatitis ${ }^{9,10}$ and in Huntington's disease. ${ }^{11} \mathrm{KMO}$ is a key regulator of metabolic flux through the kynurenine pathway of tryptophan metabolism, the main route of tryptophan metabolism in mammals. ${ }^{12}$ Perturbations in KMO activity, using genetically altered mice deficient in $\mathrm{KMO}^{12}$ and by specific inhibitors, ${ }^{12,13}$ generate biologically important alterations in plasma and tissue concentrations of several neuroactive and immunoregulatory metabolites. KMO is found in high concentrations in the liver and kidney and is also expressed by macrophages and microglia cells. ${ }^{7}$ The protein is localized on the mitochondrial outer membrane and contains a stretch of hydrophobic amino acids at its $C$ terminus, which is believed to be the mitochondrial membrane-anchoring domain. ${ }^{13}$ This transmembrane domain is thought to account for the solubility difficulties encountered during the recombinant protein production of the human enzyme. ${ }^{14}$ The crystal structure of human KMO remains as-yet unsolved in the public domain. Crystallization of membrane proteins is particularly challenging because of the need for high yields of correctly folded, homogeneous, stable protein. ${ }^{15}$ Importantly, detergent is commonly required for the solubility and stability of membrane proteins but is often detrimental to the success of crystallization attempts. ${ }^{16}$ Approaches to tackle human KMO inhibition using a Pseudomonas fluorescens KMO crystal structure have been successful, ${ }^{17,18}$ resulting in the exploration of active compound series and progression to a clinical candidate. ${ }^{19}$ In our study, we took a ligand-based ${ }^{20}$ approach, applying molecular similarity techniques to rank approved and experimental drugs in similarity to known KMO inhibitors.

\section{RESULTS AND DISCUSSION}

Ten small molecules known to be KMO inhibitors with nanomolar- to low-micromolar-range half-maximal inhibitory concentrations $\left(\mathrm{IC}_{50} \mathrm{~s}\right)$ (Supporting Information Table S1) were selected as input to molecular similarity techniques (see Experimental Section). Small-molecule similars were extracted from the DrugBank ${ }^{21}$ database. Nine of the actives were deemed too dissimilar to anything in DrugBank to follow up experimentally. One active compound, termed "Pharmacia

Received: December 30, 2017

Accepted: February 7, 2018

Published: March 2, 2018 
WO199805660 core scaffold" displayed a high degree of similarity to seven small molecules within DrugBank (Supporting Information Table S2). Patent literature documents derivatives of the Pharmacia WO199805660 core scaffold as having $\mathrm{IC}_{50} \mathrm{~s}$ of less than $100 \mu \mathrm{M}$ against Rattus norvegicus KMO. Substructure searching of the ChEMBL database ${ }^{22}$ (version 23) revealed a series of 16 derivatives that have activities on eight kinases based on an $\mathrm{IC}_{50}$ value cutoff of 10 $\mu \mathrm{M}$ (see Supporting Information Table S3). However, no targets for the unmodified Pharmacia WO199805660 core scaffold are reported in ChEMBL. We were able to purchase only four (Figure 1) of the seven priority molecules: quinaldic
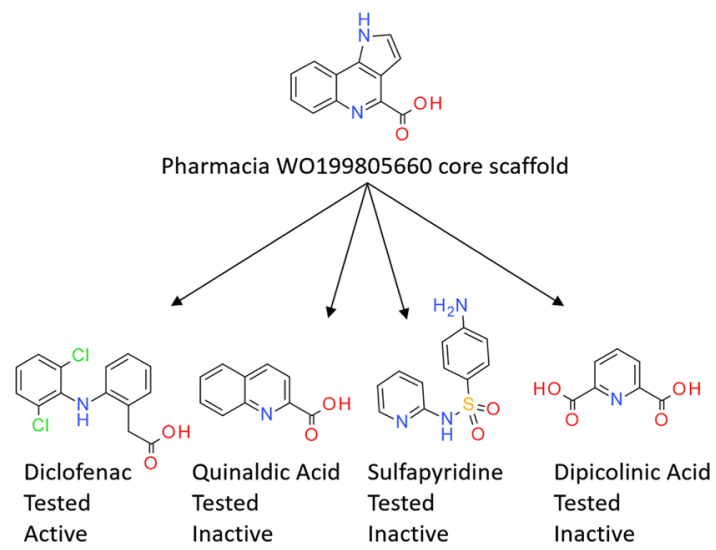

Figure 1. Molecular similarity to the Pharmacia WO199805660 core scaffold led to four compounds being sourced for testing. Only diclofenac was identified as an active inhibitor of KMO.

acid, dipicolinic acid, sulfapyridine, and diclofenac. Quinaldic acid is a product of $\mathrm{L}-\mathrm{Kyn}$ catabolism via the kynurenic acid side arm of the kynurenine pathway. ${ }^{23}$ Dipicolinic acid composes $5-15 \%$ of bacterial spores by weight and has been proposed as a key component in their heat stress pathways. ${ }^{24}$ Sulfapyridine, an antibacterial classically used to treat pneumonia and widely used during the Second World War, is now no longer widely prescribed because of the problems with crystallization in the bladder. Finally, diclofenac, a nanomolar inhibitor of cyclooxygenases, is a widely used nonsteroidal anti-inflammatory ${ }^{25}$ used to treat inflammation and pain in both humans and animals.

The four commercially available compounds were assayed in our KMO lysate enzymatic inhibition assay. ${ }^{26}$ Only diclofenac was shown to have inhibitory properties against the KMO enzyme, achieving a $38 \%$ inhibition at a diclofenac concentration of $10 \mu \mathrm{M}$. Enzymatic testing revealed an $\mathrm{IC}_{50}$ of $13.6 \pm$ $1.34 \mu \mathrm{M}$ for diclofenac against $\mathrm{KMO}$ in the cell lysate and 1.35 $\pm 0.10 \mathrm{mM}$ against whole cells, as shown by the dose-response curves in Figure 2. Our microdialysis binding assay ${ }^{27}$ demonstrated a $K_{\mathrm{D}}$ of $64.84 \pm 29.47 \mu \mathrm{M}$ for the interaction and direct binding of diclofenac to KMO (Table 1).

After the confirmation of diclofenac as a binder and inhibitor of KMO, five Saccharomyces cerevisiae KMO crystal structures were published by Amaral et al. ${ }^{28}$ Of key interest to the evaluation of diclofenac as a $\mathrm{KMO}$ inhibitor are two of these structures: first, PDB ID 4J36 containing KMO, a flavinadenine dinucleotide (FAD) molecule, and a small-molecule inhibitor and second UPF 648, a 2-(3,4-dichlorobenzoyl)cyclopropanecarboxylic acid, displaying a $K_{\mathrm{i}}$ of $74 \pm 14 \mathrm{nM}$ against yeast $\mathrm{KMO}$ and $56.7 \pm 6.8 \mathrm{nM}$ against human $\mathrm{KMO}$.

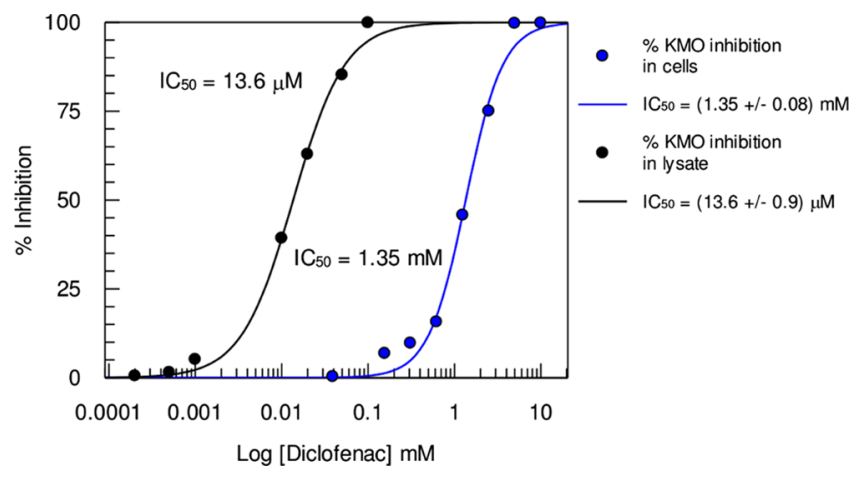

Figure 2. Plot showing the inhibition of KMO by diclofenac in whole cells and in the cell lysate-based assays.

Table 1. Summary of Diclofenac vs KMO Inhibition in the Enzymatic and Microdialysis Assays

\begin{tabular}{lcccc} 
assay & protein source & protein/assay & parameter & value \\
kinetic & $\begin{array}{c}\text { HEK-KMO cell } \\
\text { lysate }\end{array}$ & $\begin{array}{c}200 \mu \mathrm{G} \text { of total } \\
\text { protein }\end{array}$ & $\mathrm{IC}_{50}$ & $13.6 \mu \mathrm{M}$ \\
mictic & $\begin{array}{c}\text { HEK-KMO } \\
\text { whole cells } \\
\text { enriched } \\
\text { HEK-KMO } \\
\text { lysate }\end{array}$ & $\begin{array}{c}10 \mu 00 \text { cells } \\
\text { protein }\end{array}$ & $\mathrm{IC}_{50}$ & $1.35 \mathrm{mM}$ \\
& & $\mathrm{K}_{\mathrm{D}}$ & $64.8 \mu \mathrm{M}$ \\
& & & \\
\hline
\end{tabular}

Another structure with PDB ID 4J33 contains S. cerevisiae $\mathrm{KMO}$ bound to only the FAD molecule, allowing a valuable comparison of conformational changes upon inhibitor binding. Amaral et al. suggest, through the use of model building, that UPF 648 acts as a mimic of L-Kyn, binding into the same pocket, blocking L-Kyn turnover via NADPH into 3-HK. However, UPF 648 is larger than L-Kyn, with a molecular weight of 259 versus 208, and has a significantly more hydrophobic bulk ( $\log P$ values of 2.27 and -1.3 , respectively). This is structurally accounted for by loop movement (Pro321-Gln325) and reorientation of a sixstranded antiparallel $\beta$-sheet. Amaral asserts the validity for the use of S. cerevisiae KMO as a template for a structure-based drug design against human KMO. In addition, the nonhuman structure of $P$. fluorescens KMO has been used to develop compounds with nanomolar $\mathrm{IC}_{50} \mathrm{~s}^{18}$ as well as a clinical candidate. ${ }^{19}$ Visual overlays of UPF 648 and diclofenac show a striking similarity, suggesting a common binding mode. Indeed, docking of diclofenac into the S. cerevisiae KMO structure with PDB ID 4J36 using Autodock Vina 1.1.2 (see Figure 3 and Supporting Information for docking protocol) reveals a plausible binding mode.

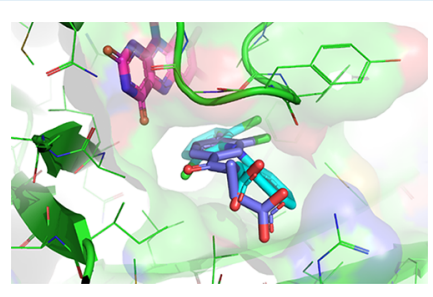

Figure 3. Saccharomyces cerevisiae KMO (green) from the structure with PDB ID 4J36 in the complex with the FAD cofactor (magenta) and UPF 648 (blue). Predicted binding mode of diclofenac (cyan) is shown in the overlay. 


\section{CONCLUSION}

In conclusion, we have successfully applied molecular similarity methods to identify diclofenac, an approved and widely used drug, as capable of binding to, and inhibiting, human KMO with a limited low micromolar affinity and activity in vitro and in cell lysate. Because of the difficulty in purifying full-length human $\mathrm{KMO}$, the binding affinity is possibly underestimated and might be closer to the lysate-derived $\mathrm{IC}_{50}$ value, with both results representing good estimates. The considerable potency reduction of $\sim 1 \log$ step of diclofenac in cells is likely due to the impaired cellular permeability associated with the carboxylic acid moiety. Additionally, diclofenac is known to bind to and inhibit many extra- and intracellular targets. Interestingly, none of the eight kinases reported as targets for the Pharmacia core scaffold are shared with diclofenac. The ChEMBL database (version 23) reports single-digit nanomolar $\mathrm{IC}_{50} \mathrm{~s} / K_{\mathrm{D}} \mathrm{s}$ for three protein targets-cyclooxygenase 1 and 2 and the interleukin- 8 receptor A. See Supporting Information Table S4 for ChEMBL-assigned targets of diclofenac. Depending on the abundance and affinities of the high number of known targets for diclofenac, the effective concentration exposed to intracellular KMO may be greatly reduced. It is important to emphasize that the presented results only highlight a starting point for a possible drug repurposing process. However, the data serve to highlight the power of molecular similarity techniques and their value in the discovery of medicinal chemistry starting points and the prediction of off-target effects. Intensive work combining structural biology and several rounds of medicinal chemistry would be needed to identify a diclofenac derivative with nanomolar activity to reach a lead compound status for KMO.

\section{EXPERIMENTAL SECTION}

Molecular Similarity. A survey of literature followed by visual inspection and clustering identified 10 potent KMO inhibitors (Supporting Information Table S1) that had their predicted lowest energy three-dimensional (3D) conformation generated using Omega2 ${ }^{29}$ (OpenEye Scientific, version 2.3.2) and written out in the SD file format. A data set containing approved, experimental, nutraceutical, illicit, and withdrawn drugs was obtained from the DrugBank ${ }^{21}$ Web service (data downloaded on 30/6/2010), and the same 3D conformer generation procedure was applied with the addition of a saltstripping step using OpenBabel ${ }^{30}$ (version 2.2.2), generating 4648 low-energy conformers. Programs implementing MACCS, Path, and LINGO fingerprints were implemented in the Java programming language using the Chemistry Development Kit. ${ }^{31} \mathrm{C}++$ programs were written making use of the OpenBabel $\mathrm{API}^{30}$ to generate FP2, FP3, and FP4 fingerprints. Implementations of USR $^{32}$ and UFSRAT ${ }^{33}$ were also written in $\mathrm{C}++$. Tanimoto distances ${ }^{34}$ were used to score molecules by fingerprint-based methods (FP2, FP3, FP4, Path, LINGO, and MACCS). A simple Euclidean distance was used for USR and UFSRAT similarity comparison scoring. Each of the 10 known KMO inhibitors was then scored against each of the 4648 drug data set using each of the 8 measures of molecular similarity, resulting in a total of 371840 similarity comparisons (see Supporting Information spreadsheet). Each similarity method run on a known inhibitor was then sorted in the order of descending level of calculated similarity. See Supporting Information for full results. Manual inspection of results revealed that only the UFSRAT similarity method applied to the Pharmacia WO199805660 core scaffold gave rise to convincing similars. Seven of these similars were prioritized, but only four were available for purchase and subsequent assay.

Cloning of full-length human KMO (KMO (V5-6His) construct), expression of this construct in HEK293 cells, and analysis of KMO enzymatic activity using liquid chromatography-mass spectrometry (LC-MS) were performed, as described by us previously. ${ }^{10}$

Compound $\mathrm{IC}_{50}$ Determination in the KMO Lysate. To determine the $\mathrm{IC}_{50}$ of compounds against human $\mathrm{KMO}$ enzyme in the cell lysate, ${ }^{10}$ the compounds were incubated in duplicate at the following concentrations: $200,100,50,20,10$, $1,0.5,0.2$, and $0.1 \mu \mathrm{M}$. The low control for this assay consisted of $200 \mu \mathrm{g}$ of cell lysate (total protein) in the absence of an inhibitor, whereas high controls were enzyme-free.

$\mathrm{IC}_{50}$ data were plotted using the GraFit v5.0.1 software (Erithacus Software Limited). The standard $\mathrm{IC}_{50}$ equation in GraFit, $y=\frac{100}{1+\mathrm{e}^{\left(s \times \ln \mid \frac{x}{\left.\left|C_{50}\right|\right)}\right)}}$, was used to fit the data to obtain $\mathrm{IC}_{50}$ and the Hill slope value $s$. Input parameters were percentage of inhibition $(y)$ and logarithm of compound concentration $(x)$.

Cellular Compound IC $\mathrm{C}_{50}$ Determination. The $\mathrm{IC}_{50}$ values of compounds were tested in the cellular context to determine the cell permeability of test compounds. HEK293-KMO(V5$6 \mathrm{His})$ cells were passaged in 96-well plates at $2 \times 10^{4}$ cells per well in Dulbecco's modified Eagle's medium with $10 \%$ fetal bovine serum, $1 \%$ L-glutamine, and $1 \%$ penicillin-streptomycin. The cells were incubated overnight at $37{ }^{\circ} \mathrm{C}$ in $5 \% \mathrm{CO}_{2} /$ $95 \% \mathrm{O}_{2}$. The assay medium utilized was opti-MEM containing $1 \%$ L-glutamine, $1 \%$ penicillin-streptomycin, and $200 \mu \mathrm{M} \mathrm{L}$ Kyn. The compounds for testing were diluted in this medium with a final dimethyl sulfoxide (DMSO) concentration of $1 \%$. $3-\mathrm{HK}$ was diluted in the assay medium in the absence of L-Kyn to produce a standard curve with the following concentrations: $200,100,50,20,10,1,0.5,0.2$, and $0.1 \mu \mathrm{M}$. Low controls consisted of opti-MEM with $1 \%$ L-glutamine, $1 \%$ penicillinstreptomycin, $200 \mu \mathrm{M}$ L-Kyn, and $1 \%$ DMSO, whereas high controls consisted of the same buffer minus L-Kyn. The standards, compounds, and control dilutions were added to the cells over $20 \mathrm{~h}$ at $37{ }^{\circ} \mathrm{C}$ in $5 \% \mathrm{CO}_{2} / 95 \% \mathrm{O}_{2}$. Following incubation, the assay samples (i.e., cellular supernatants) were transferred to a 96-well masterblock before the addition of 500 $\mu \mathrm{L}$ of acetonitrile containing $25 \mu \mathrm{g} / \mathrm{mL}$ d5-tryptophan per well to terminate activity. This solution was dried under nitrogen, and each sample was solubilized by the addition of $100 \mu \mathrm{L}$ of 30:70 methanol/water with $0.1 \%$ formic acid. The samples were transferred to 96-well v-bottom plates for MS analysis. (MS analysis was performed as described above.)

Microdialysis. Microdialysis was used to assess the direct binding affinity, expressed as dissociation constant, $K_{\mathrm{D}}$, of diclofenac for $\mathrm{KMO}$ in a method similar to that reported by Weidemann et al. ${ }^{27}$ KMO (expressed as above) was enriched by NiNTA affinity chromatography. RED (rapid equilibrium dialysis) device inserts (molecular weight cutoff $8 \mathrm{kDa}$ ) were placed in the wells of a compatible base plate (Piece, Thermo Scientific). The enriched KMO target protein was diluted to a concentration of $10 \mu \mathrm{M}$ in the assay buffer [20 mM 4-(2hydroxyethyl)-1-piperazineethanesulfonic acid $\mathrm{pH}$ 7.0] in a total volume of $100 \mu \mathrm{L}$ and added to the sample chamber of the RED device insert. Assay buffer $(300 \mu \mathrm{L})$ was added to the dialysis chamber of the RED device insert. The compound was added to the starter volume of the sample chamber at a final concentration of $20 \mu \mathrm{M}$. Controls consisted of compounds at 
the same concentration in the assay buffer in the absence of the enzyme. The plate was covered with a sealing tape and incubated for $6 \mathrm{~h}$ at $37^{\circ} \mathrm{C}$ with rotation at $100 \mathrm{rpm}$. Samples of $50 \mu \mathrm{L}$ were obtained from both the sample and dialysis compartments of the chamber and transferred to LC-MS vials with $10 \mu \mathrm{L}$ of acetonitrile per sample for MS analysis (see Supporting Information). $K_{\mathrm{D}}$ values were calculated using the data obtained from MS analysis according to the formula determined by Weidemann et al. ${ }^{27}$ Diclofenac was diluted in the assay buffer at the concentrations of $1,2,5,10,20$, and 50 $\mu \mathrm{M}$ in LC-MS vials to provide a standard curve, allowing quantification of concentrations from the assay samples. The samples were analyzed using the MS method previously described. $^{35}$

\section{ASSOCIATED CONTENT}

\section{S Supporting Information}

The Supporting Information is available free of charge on the ACS Publications website at DOI: 10.1021/acsomega.7b02091.

Molecular similarity results (XLSX)

Known KMO inhibitors used as input to molecular similarity techniques; prioritized drugs for assay; and AutoDock Vina docking protocol and parameters (PDF)

\section{AUTHOR INFORMATION}

\section{Corresponding Author}

*E-mail: manfred.auer@ed.ac.uk (M.A.).

\section{ORCID 1}

Manfred Auer: 0000-0001-8920-3522

\section{Author Contributions}

S.S. and K.M. contributed equally to this work.

\section{Notes}

The authors declare no competing financial interest.

\section{ACKNOWLEDGMENTS}

The authors acknowledge financial support from the Scottish Universities Life Sciences Alliance ((SULSA-http://www. sulsa.ac.uk), the Medical Research Council ((MRC-www.mrc. ac.uk, J54359), the Strategic Grant, and the European Community's 7th Framework Program (FP7/2007-2013) under grant agreement no 278568 "PRIMES" (M.A., S.S.). D.J.M. acknowledges the support of The Health Foundation through a Clinician Scientist Fellowship at the time this work was done and is currently supported by an MRC Senior Clinical Fellowship. In addition, we would like to thank Prof. Malcolm Walkinshaw and Dr. Paul Taylor for access to computational resources and algorithms used in the creation of the UFSRAT molecular similarity technique.

\section{REFERENCES}

(1) DiMasi, J. A.; Grabowski, H. G.; Hansen, R. W. Innovation in the pharmaceutical industry: New estimates of R\&D costs. J. Health Econ. 2016, 47, 20-33.

(2) Waring, M. J.; Arrowsmith, J.; Leach, A. R.; Leeson, P. D.; Mandrell, S.; Owen, R. M.; Pairaudeau, G.; Pennie, W. D.; Pickett, S. D.; Wang, J.; Wallace, O.; Weir, A. An analysis of the attrition of drug candidates from four major pharmaceutical companies. Nat. Rev. Drug Discovery 2015, 14, 475-486.

(3) Cha, Y.; Erez, T.; Reynolds, I. J.; Kumar, D.; Ross, J.; Koytiger, G.; Kusko, R.; Zeskind, B.; Risso, S.; Kagan, E.; Papapetropoulos, S.; Grossman, I.; Laifenfeld, D. Drug repurposing from the perspective of pharmaceutical companies. Br. J. Pharmacol. 2017, 175, 168-180.
(4) Xu, M.; Lee, E. M.; Wen, Z.; Cheng, Y.; Huang, W.-K.; Qian, X.; Tcw, J.; Kouznetsova, J.; Ogden, S. C.; Hammack, C.; Jacob, F.; Nguyen, H. N.; Itkin, M.; Hanna, C.; Shinn, P.; Allen, C.; Michael, S. G.; Simeonov, A.; Huang, W.; Christian, K. M.; Goate, A.; Brennand, K. J.; Huang, R.; Xia, M.; Ming, G.-L.; Zheng, W.; Song, H.; Tang, H. Identification of small-molecule inhibitors of Zika virus infection and induced neural cell death via a drug repurposing screen. Nat. Med. 2016, 22, 1101-1107.

(5) Conroy, S.; McIntyre, J.; Choonara, I. Unlicensed and off label drug use in neonates. Arch. Dis. Child. Fetal Neonatal Ed. 1999, 80, F142-F145.

(6) Choonara, I.; Conroy, S. Unlicensed and off-label drug use in children. Drug Saf. 2002, 25, 1-5.

(7) Okuda, S.; Nishiyama, N.; Saito, H.; Katsuki, H. 3Hydroxykynurenine, an endogenous oxidative stress generator, causes neuronal cell death with apoptotic features and region selectivity. $J$. Neurochem. 2002, 70, 299-307.

(8) Wilson, K.; Auer, M.; Binnie, M.; Zheng, X.; Pham, N. T.; Iredale, J. P.; Webster, S. P.; Mole, D. J. Overexpression of human kynurenine3-monooxygenase protects against 3-hydroxykynurenine-mediated apoptosis through bidirectional nonlinear feedback. Cell Death Dis. 2016, 7, No. e2197.

(9) Skouras, C.; Zheng, X.; Binnie, M.; Homer, N. Z. M.; Murray, T. B. J.; Robertson, D.; Briody, L.; Paterson, F.; Spence, H.; Derr, L. Increased levels of 3-hydroxykynurenine parallel disease severity in human acute pancreatitis. Sci. Rep. 2016, 6, 33951.

(10) Mole, D. J.; Webster, S. P.; Uings, I.; Zheng, X.; Binnie, M.; Wilson, K.; Hutchinson, J. P.; Mirguet, O.; Walker, A.; Beaufils, B. Kynurenine-3-monooxygenase inhibition prevents multiple organ failure in rodent models of acute pancreatitis. Nat. Med. 2016, 22, 202.

(11) Giorgini, F.; Guidetti, P.; Nguyen, Q.; Bennett, S. C.; Muchowski, P. J. A genomic screen in yeast implicates kynurenine 3-monooxygenase as a therapeutic target for Huntington disease. Nat. Genet. 2005, 37, 526-531.

(12) Moffett, J. R.; Namboodiri, M. A. A. Tryptophan and the immune response. Immunol. Cell Biol. 2003, 81, 247-265.

(13) Okamoto, H.; Yamamoto, S.; Nozaki, M.; Hayaishi, O. On the submitochondrial localization of 1-kynurenine-3-hydroxylase. Biochem. Biophys. Res. Commun. 1967, 26, 309-314.

(14) Breton, J.; Avanzi, N.; Magagnin, S.; Covini, N.; Magistrelli, G.; Cozzi, L.; Isacchi, A. Functional characterization and mechanism of action of recombinant human kynurenine 3-hydroxylase. Eur. J. Biochem. 2000, 267, 1092-1099.

(15) Garavito, R. M.; Picot, D.; Loll, P. J. Strategies for crystallizing membrane proteins. J. Bioenerg. Biomembr. 1996, 28, 13-27.

(16) Prince, C.; Jia, Z. Measurement of detergent concentration using 2, 6-dimethylphenol in membrane-protein crystallization. Acta Crystallogr., Sect. D: Biol. Crystallogr. 2012, 68, 1694-1696.

(17) Hutchinson, J. P.; Rowland, P.; Taylor, M. R. D.; Christodoulou, E. M.; Haslam, C.; Hobbs, C. I.; Holmes, D. S.; Homes, P.; Liddle, J.; Mole, D. J.; Uings, I.; Walker, A. L.; Webster, S. P.; Mowat, C. G.; Chung, C.-W. Structural and mechanistic basis of differentiated inhibitors of the acute pancreatitis target kynurenine-3-monooxygenase. Nat. Commun. 2017, 8, 15827.

(18) Liddle, J.; Beaufils, B.; Binnie, M.; Bouillot, A.; Denis, A. A.; Hann, M. M.; Haslam, C. P.; Holmes, D. S.; Hutchinson, J. P.; Kranz, M.; McBride, A.; Mirguet, O.; Mole, D. J.; Mowat, C. G.; Pal, S.; Rowland, P.; Trottet, L.; Uings, I. J.; Walker, A. L.; Webster, S. P. The discovery of potent and selective kynurenine 3-monooxygenase inhibitors for the treatment of acute pancreatitis. Bioorg. Med. Chem. Lett. 2017, 27, 2023-2028.

(19) Walker, A. L.; Ancellin, N.; Beaufils, B.; Bergeal, M.; Binnie, M.; Bouillot, A.; Clapham, D.; Denis, A.; Haslam, C. P.; Holmes, D. S.; Hutchinson, J. P.; Liddle, J.; McBride, A.; Mirguet, O.; Mowat, C. G.; Rowland, P.; Tiberghien, N.; Trottet, L.; Uings, I.; Webster, S. P.; Zheng, X.; Mole, D. J. Development of a Series of Kynurenine 3Monooxygenase Inhibitors Leading to a Clinical Candidate for the Treatment of Acute Pancreatitis. J. Med. Chem. 2017, 60, 3383-3404. 
(20) Geppert, H.; Vogt, M.; Bajorath, J. Current trends in ligandbased virtual screening: molecular representations, data mining methods, new application areas, and performance evaluation. $J$. Chem. Inf. Model. 2010, 50, 205-216.

(21) Wishart, D. S.; Knox, C.; Guo, A. C.; Cheng, D.; Shrivastava, S.; Tzur, D.; Gautam, B.; Hassanali, M. DrugBank: a knowledgebase for drugs, drug actions and drug targets. Nucleic Acids Res. 2008, 36, D901-D906.

(22) Gaulton, A.; Bellis, L. J.; Bento, A. P.; Chambers, J.; Davies, M.; Hersey, A.; Light, Y.; McGlinchey, S.; Michalovich, D.; Al-Lazikani, B.; Overington, J. P. ChEMBL: a large-scale bioactivity database for drug discovery. Nucleic Acids Res. 2012, 40, D1100-D1107.

(23) Takahashi, H.; Kaihara, M.; Price, J. The conversion of kynurenic acid to quinaldic acid by humans and rats. J. Biol. Chem. 1956, 223, 705-708.

(24) Slieman, T. A.; Nicholson, W. L. Role of Dipicolinic Acid in Survival ofBacillus subtilis Spores Exposed to Artificial and Solar UV radiation. Appl. Environ. Microbiol. 2001, 67, 1274-1279.

(25) Todd, P. A.; Sorkin, E. M. Diclofenac sodium. Drugs 1988, 35, 244-285.

(26) Lowe, D. M.; Gee, M.; Haslam, C.; Leavens, B.; Christodoulou, E.; Hissey, P.; Hardwicke, P.; Argyrou, A.; Webster, S. P.; Mole, D. J.; Wilson, K.; Binnie, M.; Yard, B. A.; Dean, T.; Liddle, J.; Uings, I.; Hutchinson, J. P. Lead discovery for human kynurenine 3monooxygenase by high-throughput RapidFire mass spectrometry. J. Biomol. Screening 2014, 19, 508-515.

(27) Weidemann, T.; Seifert, J.-M.; Hintersteiner, M.; Auer, M. Analysis of protein-small molecule interactions by microscale equilibrium dialysis and its application as a secondary confirmation method for on-bead screening. J. Comb. Chem. 2010, 12, 647-654.

(28) Amaral, M.; Levy, C.; Heyes, D. J.; Lafite, P.; Outeiro, T. F.; Giorgini, F.; Leys, D.; Scrutton, N. S. Structural basis of kynurenine 3monooxygenase inhibition. Nature 2013, 496, 382.

(29) Hawkins, P. C. D.; Skillman, A. G.; Warren, G. L.; Ellingson, B. A.; Stahl, M. T. Conformer generation with OMEGA: algorithm and validation using high quality structures from the Protein Databank and Cambridge Structural Database. J. Chem. Inf. Model. 2010, 50, 572584.

(30) O'Boyle, N. M.; Banck, M.; James, C. A.; Morley, C.; Vandermeersch, T.; Hutchison, G. R. Open Babel: An open chemical toolbox. J. Cheminf. 2011, 3, 33.

(31) Steinbeck, C.; Han, Y.; Kuhn, S.; Horlacher, O.; Luttmann, E.; Willighagen, E. The Chemistry Development Kit (CDK): An opensource Java library for chemo-and bioinformatics. J. Chem. Inf. Comput. Sci. 2003, 43, 493-500.

(32) Ballester, P. J.; Richards, W. G. Ultrafast shape recognition to search compound databases for similar molecular shapes. J. Comput. Chem. 2007, 28, 1711-1723.

(33) Shave, S.; Blackburn, E. A.; Adie, J.; Houston, D. R.; Auer, M.; Webster, S. P.; Taylor, P.; Walkinshaw, M. D. UFSRAT: Ultra-Fast Shape Recognition with Atom Types-The Discovery of Novel Bioactive Small Molecular Scaffolds for FKBP12 and 11 $\beta$ HSD1. PLoS One 2015, 10, No. e0116570.

(34) Lipkus, A. H. A proof of the triangle inequality for the Tanimoto distance. J. Math. Chem. 1999, 26, 263-265.

(35) Wilson, K.; Mole, D. J.; Homer, N. Z. M.; Iredale, J. P.; Auer, M.; Webster, S. P. A magnetic bead-based ligand binding assay to facilitate human kynurenine 3-monooxygenase drug discovery. $J$. Biomol. Screening 2015, 20, 292-298. 